\title{
An Enhanced Tree-shaped Adachi-like Chaotic Neural Network Requiring Linear-time Computations
}

\author{
Ke Qin ${ }^{1 \star}$ and B. John Oommen ${ }^{2 \star \star}$ \\ 1 School of Computer Science and Engineering \\ University of Electronic Science \& Technology of China \\ 610054, Chengdu, China \\ (e-mail: thinker_qk@hotmail.com) \\ 2 School of Computer Science \\ Carleton University \\ K1S 5B6, Ottawa, ON, Canada \\ (e-mail: oommen@scs.carleton.ca)
}

\begin{abstract}
The Adachi Neural Network (AdNN) [1-5], is a fascinating Neural Network (NN) which has been shown to possess chaotic properties, and to also demonstrate Associative Memory (AM) and Pattern Recognition (PR) characteristics. Variants of the AdNN [6,7] have also been used to obtain other PR phenomena, and even blurring. A significant problem associated with the AdNN and its variants, is that all of them require a quadratic number of computations. This is essentially because all their NNs are completely connected graphs. In this paper we consider how the computations can be significantly reduced by merely using a linear number of computations. To do this, we extract from the original complete graph, one of its spanning trees. We then compute the weights for this spanning tree in such a manner that the modified tree-based NN has approximately the same input-output characteristics, and thus the new weights are themselves calculated using a gradient-based algorithm. By a detailed experimental analysis, we show that the new linear-time AdNN-like network possesses chaotic and PR properties for different settings. As far as we know, such a tree-based AdNN has not been reported, and the results given here are novel.
\end{abstract}

Keywords: Chaotic Neural Networks, Chaotic Pattern Recognition, Adachi Neural Network.

\section{Introduction}

Artificial Neural Network (ANN) is one of the four best approaches for PR. ANN attempts to use some organizational principles such as learning, gen-

* The work of this author was done while he was in Canada as a Visiting Scholar at Carleton University in 2008. The author gratefully acknowledges the support of the K. C. Wong Education Foundation of Hong Kong.

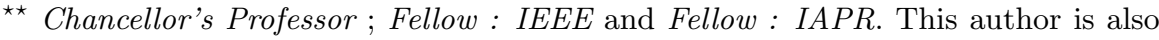
an Adjunct Professor with the University of Agder in Grimstad, Norway. The work of this author was partially supported by NSERC, the Natural Sciences and Engineering Research Council of Canada. 
eralization, adaptivity, fault tolerance, distributed representation, and computation in order to achieve the recognition. One of the limitations of most ANN models is the dependency on an external stimulation. Once an output pattern has been identified, the ANN remains in that state until the arrival of a new external input. This is in contrast to real biological NNs which exhibit sequential memory characteristics. To be more specific, once a pattern is recalled from a memory location, the brain is not "stuck" to it, it is also capable of recalling other associated memory patterns without being prompted by any additional external inputs. This ability to "jump" from one memory state to another in the absence of a stimulus is one of the hallmarks of the brain, and this is one phenomenon that a chaotic PR system has to emulate.

The goal of the field of Chaotic PR systems can be expressed as follows: We do not intend a chaotic PR system to report the identity of a testing pattern with such a "proclamation". Rather, what we want to achieve, on one hand, is to have the chaotic PR system give a strong periodic or more frequent signal when a pattern is to be recognized. Further, between two consecutive recognized patterns, none of the trained patterns must be recalled. Finally, and most importantly, if an untrained pattern is presented, the system must give a chaotic signal.

This paper deals with the Adachi Neural Network AdNN [1-5], which possesses a spectrum of very interesting chaotic, AM and PR properties, as described in $[6,7]$. The fundamental "problem" associated with the AdNN and its variants is its quadratic computational requirement. We shall show that by using spanning tree concepts and an effective gradient search strategy, this burden can be reduced to be linear, and yet be almost as effective with regard to the chaotic and PR characteristics.

\section{Limitations of the Current Schemes}

Although the works of Adachi et al and Calitoiu et al were ground-breaking, it turns out that, as stated in [9], the results claimed in the prior $\operatorname{art}^{1}$ were not as precise as stated. Apart from this limitation, the computational burden is excessive, rendering it as an impractical machine. More precisely, we mention the following "drawbacks" of the state-of-the-art:

1. The AdNN's power as a PR system requires excessive computations of the order of $O\left(N^{2}\right)$. For the examples cited by Adachi et al and Calitoiu et al [1-7], which use $10 \times 10$ pixel arrays, this involves 10,000 computations per time step.

2. The PR capabilities of the AdNN are limited, as will be explained presently. Our intention is to enhance these.

\footnotetext{
${ }^{1}$ The state of the art of the AdNN and its variants can be found in $[6,7,9]$.
} 
3. It is also appropriate that the PR limitations of the work of Calitoiu et al [7] are mentioned. Although the experimental results cited in [7] display a reduction in the transient phase (when compared to the AdNN), unfortunately, the M-AdNN does not achieve PR for untrained patterns. It turns out that although unreported in [7], the M-AdNN always reproduces the input patterns periodically independent of whether the input is a trained or an untrained pattern. This has also been illustrated in [9].

\section{Designing the L-AdNN}

\subsection{The Topology of the L-AdNN}

To minimize the computational burden of the AdNN, we shall first arrive at a topology with a linear number of edges. We arrive at the modified linear version in two steps. First of all, we reduce the number of edges to be linear by using spanning tree. The second step will involve the computation of the weights associated with this new structure, which we will address subsequently. Of course, the details of these steps cannot be presented here due to space limitations, but they can be found in [8].

Since the network topology has been changed and we want the L-AdNN to maximally approximate the original AdNN, we propose to use the Maximum Spanning Tree (MST) of the AdNN (with the edge weights given by their absolute values) to be the initial linear approximation to the completelyconnected AdNN. This is formalized by Algorithm Topology_L-AdNN below. Unlike the AdNN and its variants, this initial approximation of the L-AdNN

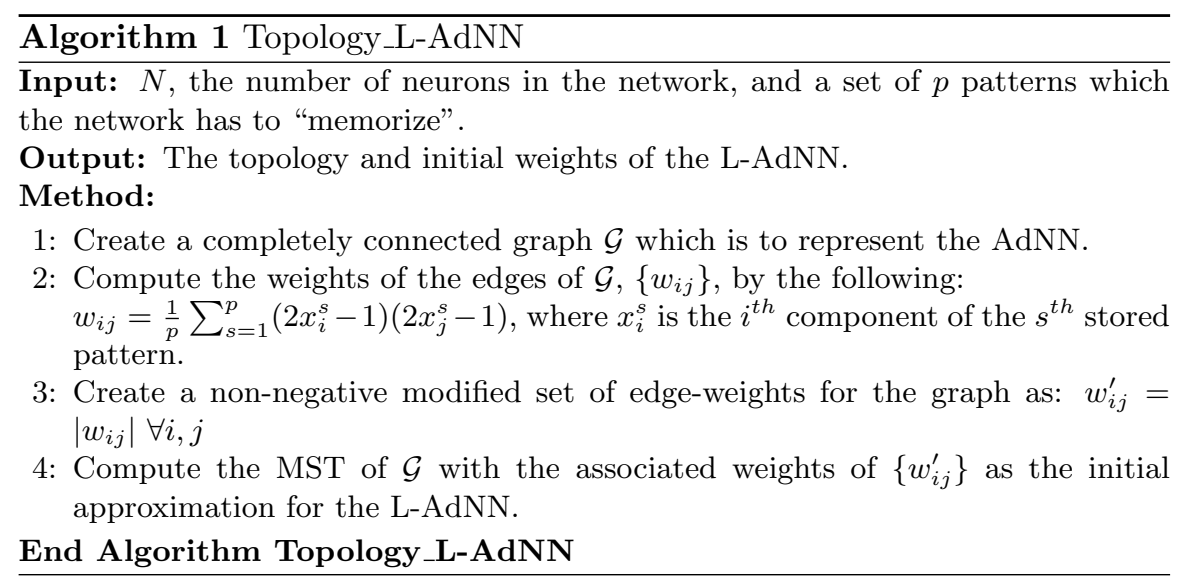

is tree-shaped, since we have pruned the edges which we believe are the most "redundant". In this regard, since that the original AdNN has $N$ neurons and $N^{2}$ edges, we recommend the use of the Kruskal algorithm which is much more efficient than the Prim algorithm in such a setting. 


\subsection{The Weights of the L-AdNN: Gradient Search}

Since we have removed most of the "redundant" edges from the completelyconnected graph by using a MST, it is clear that the NN at hand will not adequately compare with the original AdNN. Thus, our next task is to determine a new set of weights so as to force the L-AdNN to retain some of its PR properties, namely those corresponding to the trained patterns. We briefly explain below (for more details the reader is requested to refer to [8]) the process for achieving this.

The L-AdNN is defined by following equations:

$$
\begin{gathered}
x_{i}^{L}(t+1)=f\left(\eta_{i}^{L}(t+1)+\xi_{i}^{L}(t+1)\right), \\
\eta_{i}^{L}(t+1)=k_{f} \eta_{i}^{L}(t)+\sum_{e_{i j} \in T} w_{i j}^{L^{*}} x_{j}^{L}(t), \\
\xi_{i}^{L}(t+1)=k_{r} \xi_{i}^{L}(t)-\alpha x_{i}^{L}(t)+a_{i} .
\end{gathered}
$$

where $\left\{w_{i j}^{L^{*}}\right\}, x_{i}^{L}, \xi_{i}^{L}$ and $\eta_{i}^{L}$ are the weights, outputs, and state variables of the L-AdNN respectively, and have similar meanings to $\left\{w_{i j}\right\}, x_{i}, \xi_{i}$ and $\eta_{i}$ of the AdNN.

In order to find the optimal values of $\left\{w_{i j}^{L^{*}}\right\}$, we define the square error between the original output of the AdNN and new output at the $n^{\text {th }}$ step:

$$
E_{p}=\frac{1}{2} \sum_{k=1}^{N}\left(x_{i}^{A, p}-x_{i}^{L, p}(n)\right)^{2},
$$

where $x_{i}^{A, p}$ and $x_{i}^{L, p}$ imply the outputs of the $i^{\text {th }}$ neuron when the $p^{\text {th }}$ pattern is presented to the AdNN network and the L-AdNN network respectively. The overall global error is defined by $E=\sum_{p=1}^{P} E_{p}$ where $P$ is the number of trained patterns.

In order to adjust $w_{i j}^{L}$ to obtain the least global error $E$, we consider the gradient, $\Delta w_{i j}^{L}$, and move $w_{i j}^{L}$ by an amount which equals $\Delta w_{i j}^{L}$ in the direction where the error is minimized. This can be formalized as below:

$$
\begin{aligned}
\Delta w_{i j}^{L} & =-\beta \frac{\partial E}{\partial w_{i j}^{L}}=-\beta \frac{\partial \sum_{p=1}^{P} E_{p}}{\partial w_{i j}^{L}}=-\beta \sum_{p=1}^{P} \frac{\partial E_{p}}{\partial x_{i}^{L, p}(n)} \cdot \frac{\partial x_{i}^{L, p}(n)}{\partial w_{i j}^{L}} \\
& =\beta \sum_{p=1}^{P}\left(x_{i}^{A, p}-x_{i}^{L, p}(n)\right) \cdot \frac{1}{\varepsilon} \cdot x_{i}^{L, p}(n) \cdot\left(1-x_{i}^{L, p}(n)\right) \cdot x_{j}^{L, p}(n),
\end{aligned}
$$

where $\beta$ is the learning rate of the gradient search. The formal algorithm which achieves the update is given in Algorithm 2. The results of a typical numerical experiment which proceeds along the above gradient search are shown in Figure 1. In these simulations, we have chosen the learning rate $\beta$ to be 0.05 . The reader will observe that the global error, $E$, converges very 
rapidly. Indeed, after merely 60 iterations, the average value of the matrix of $\Delta w_{i j}^{L}$ converges to a value arbitrarily close to 0 (from the perspective of the machine's accuracy), and becomes invariant, thereafter.
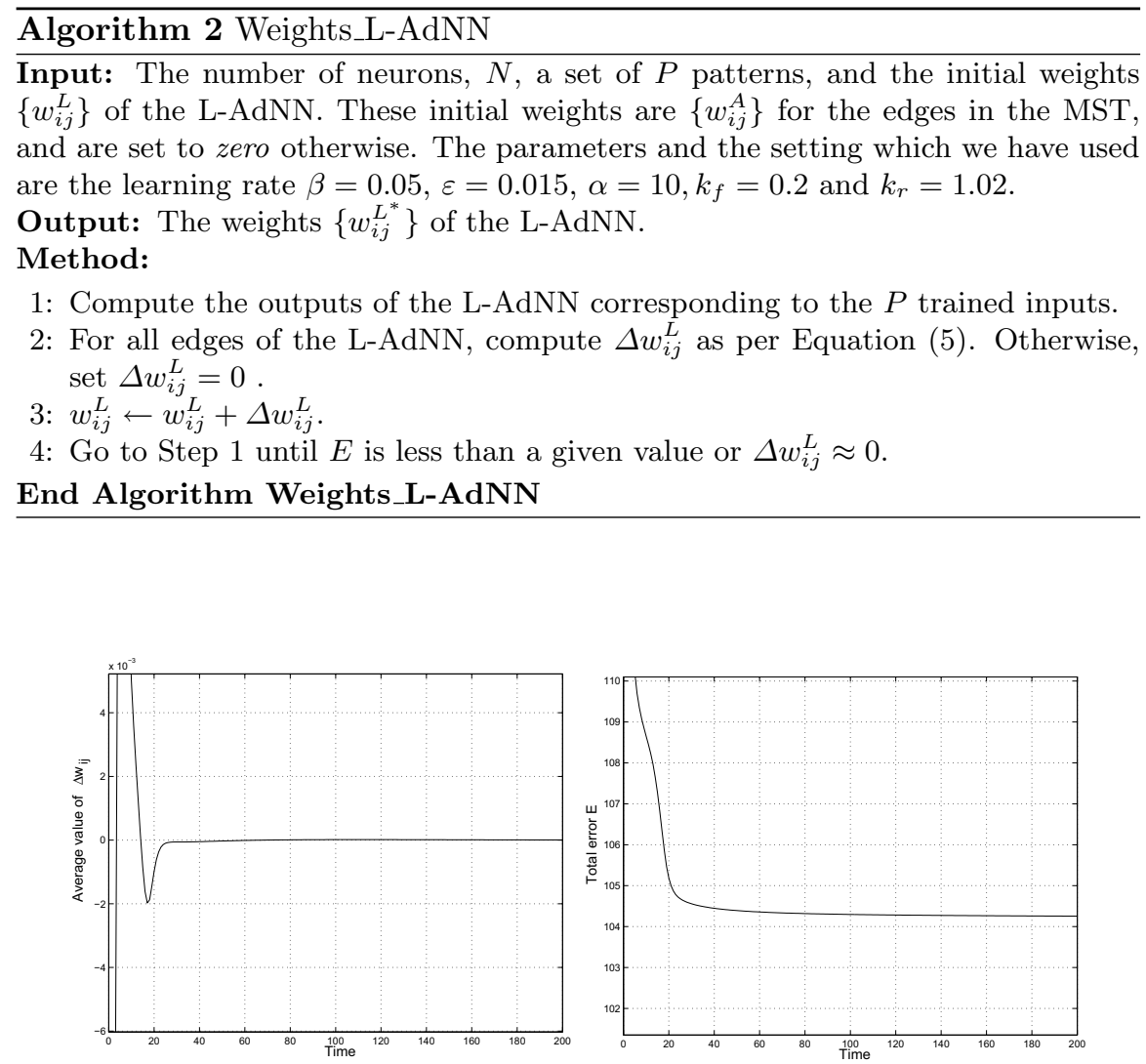

Fig. 1. The figure on the left shows the variation of the average of $\Delta w_{i j}^{L}$ (averaged over all values of $i$ and $j$ ) over the first 200 iterations of the gradient search scheme. The average converges to a value arbitrarily close to zero after 60 time steps. The figure on the right shows the variation of the global error over the same time frame. Observe that this quantity does not converge to zero.

\section{Lyapunov Exponents Analysis of the L-AdNN}

We shall now briefly submit a Lyapunov analysis of the L-AdNN. Due to space considerations, many of the details are omitted here, but are in [8].

For a dynamical system, its sensitivity to the initial conditions is quantified by its so-called Lyapunov exponents. Although the calculation of the 
Lyapunov exponents by directly using a Jacobian analysis is not feasible. However, the specific topology and state update equations of the L-AdNN makes the task feasible, as we shall presently see.

Considering the Jacobian matrix $J$ of the L-AdNN whose structure, topology and weights have been determined in Section $3 . J$ can be seen to have the form:

$$
J=\left(\begin{array}{l}
{\left[J_{i j}^{1}\right]\left[J_{i j}^{2}\right]} \\
{\left[J_{i j}^{3}\right]\left[J_{i j}^{4}\right]}
\end{array}\right)
$$

where each $\left[J_{i j}^{k}\right]$ is an $N \times N$ sub-matrix of $J$, for $1 \leq k \leq 4,1 \leq i \leq N$ and $1 \leq j \leq N$. Each $\left[J_{i j}^{k}\right]$ is a result of taking the partial derivatives of $\eta_{i}^{L}(t+1)$ and $\xi_{i}^{L}(t+1)$ with regard to $\eta_{j}^{L}(t)$ and $\xi_{j}^{L}(t)$ respectively as: $J_{i j}^{1}(t)=\frac{\partial \eta_{i}^{L}(t+1)}{\partial \eta_{j}^{L}(t)}$, $J_{i j}^{2}(t)=\frac{\partial \eta_{i}^{L}(t+1)}{\partial \xi_{j}^{L}(t)}, J_{i j}^{3}(t)=\frac{\partial \xi_{i}^{L}(t+1)}{\partial \eta_{j}^{L}(t)}, J_{i j}^{4}(t)=\frac{\partial \xi_{i}^{L}(t+1)}{\partial \xi_{j}^{L}(t)}$, for all $1 \leq i \leq N$ and $1 \leq j \leq N$

By taking the partial derivatives after considering the explicit forms of the state space equations given by $(1)-(3)$, we see that the following closed form expressions for $J_{i j}^{k}(t)$ result: (a): $J_{i j}^{1}(t)=k_{f}+\frac{w_{i j}^{L^{*}}}{\varepsilon} \cdot x_{j}^{L}(t)\left(1-x_{j}^{L}(t)\right)$ when $i=j$; (b): $J_{i j}^{1}(t)=\frac{w_{i j}^{L^{*}}}{\varepsilon} \cdot x_{j}^{L}(t)\left(1-x_{j}^{L}(t)\right)$ when $i \neq j ;(\mathrm{c}): J_{i j}^{2}(t)=$ $\frac{w_{i j}^{L^{*}}}{\varepsilon} x_{j}^{L}(t)\left(1-x_{j}^{L}(t)\right) ;(\mathrm{d}): J_{i j}^{3}(t)=-\frac{\alpha}{\varepsilon} x_{j}^{L}(t)\left(1-x_{j}^{L}(t)\right)$ when $i=j ;(\mathrm{e})$ : $J_{i j}^{3}(t)=0$ when $i \neq j$; (f): $J_{i j}^{4}(t)=k_{r}-\frac{\alpha}{\varepsilon} x_{j}^{L}(t)\left(1-x_{j}^{L}(t)\right)$ when $i=j$; (g): $J_{i j}^{4}(t)=0$ when $i \neq j$.

To get a better insight of the dynamics, we observe, first of all, the term $x_{i}^{L}(t)\left(1-x_{i}^{L}(t)\right)$ has an asymptotic value of zero for all $i$. Secondly, since the topology of the L-AdNN is tree-shaped, $w_{i j}^{L^{*}}$ should be 0 if $e_{i j}$ is not in $T$. Further, since the spanning tree topology does not contain any circles, this implies $w_{i j}^{L^{*}}=0$ for all $j=i$. Thus, the Jacobian matrix has the form:

$$
J=\left(\left[\begin{array}{cccccccc}
k_{f} & 0 & \cdots & 0 & 0 & \cdots & \cdots & 0 \\
0 & \ddots & \cdots & 0 & \vdots & \ddots & \cdots & \vdots \\
\vdots & \vdots & \ddots & \vdots & \vdots & \vdots & \ddots & \vdots \\
0 & \cdots & \cdots & \left.J_{i j}^{3}\right]\left[J_{i j}^{2}\right] & 0 & \vdots & \cdots & 0 \\
0 & \cdots & \cdots & 0 & k_{r} & 0 & \cdots & 0 \\
\vdots & \ddots & \cdots & \vdots & 0 & \ddots & \cdots & 0 \\
\vdots & \vdots & \ddots & \vdots & \vdots & \vdots & \ddots & \vdots \\
0 & \vdots & \cdots & 0 & 0 & \cdots & \cdots & k_{r}
\end{array}\right) .\right.
$$

Consequently, $J\left(x_{0}\right)=J\left(x_{1}\right)=\cdots=J\left(x_{k}\right)$. The Lyapunov exponents can be calculated by the eigenvalues of matrix $L\left(x_{0}\right)$,

$$
L\left(x_{0}\right)=\lim _{k \rightarrow \infty}\left[J^{(k)}\left(J^{(k)}\right)^{\mathrm{T}}\right]^{1 / 2 k}=\lim _{k \rightarrow \infty}\left[J^{k}\left(J^{\mathrm{T}}\right)^{k}\right]^{1 / 2 k}=J .
$$


Observe that this matrix has $2 N$ eigenvalues. thus the Lyapunov exponents are: $\lambda_{1}=\lambda_{2}=\cdots=\lambda_{N}=\log k_{f}, \lambda_{N+1}=\lambda_{N+2}=\cdots=\lambda_{2 N}=\log k_{r}$. By choosing the values of the coefficient as $k_{f}=0.2, k_{r}=1.02$, we can force the LLE to be $\lambda_{N+1}=\lambda_{N+2}=\cdots=\lambda_{2 N}=\log 1.02>0$, rendering the L-AdNN to be chaotic.

\section{Chaotic and PR Properties of the AdNNs}

We now briefly report the comparative PR properties of the L-AdNN and AdNN, and refer the reader to [8] for more detailed results. These properties have been gleaned as a result of examining the Hamming distance between the input pattern and the patterns that appear in the output.

\subsection{Comparative Chaotic Properties}

In the ideal setting we would have preferred the L-AdNN to be chaotic when exposed to untrained patterns, and the output to appear periodically or more frequently when exposed to trained patterns. Besides yielding this phenomenon, the L-AdNN also goes through a chaotic phase and a PR phase as some of its parameters change. We summarize the results for the L-AdNN

\section{XNOLS}

Fig. 2. The set of patterns used in Adachi et al's and our experiments. The first four patterns constitute the set used by Adachi et al. Pattern 5 is an untrained pattern, which represents the digit 5 .

by stating that by using different settings of $\alpha$ and $a_{i}$, the latter demonstrates the following amazing result in Figure 3. From this Figure we see that if the L-AdNN is presented with a trained pattern, the output is the same trained pattern occurring Frequently. But the output is Chaotic for untrained patterns. While this phenomenon is not observed when $a_{i}=2$ (when the L-AdNN is always Chaotic), the Frequent behaviour is noticeable when $a_{i}=2+6 x_{i}$.

Since a finer measure of how closely the output mimics the input is their Hamming distance (which is exactly zero if the output is an precise replica of the input), we have also tabulated the frequency of the Hamming distance within the first 1,000 time intervals in Table 1 for the various input patterns. Table 1 demonstrates that this distance is close to zero (i.e., between 0 and 5) quite infrequently, implying that under these settings, the L-AdNN is chaotic. It is worth mentioning that most of the Hamming distances are in 

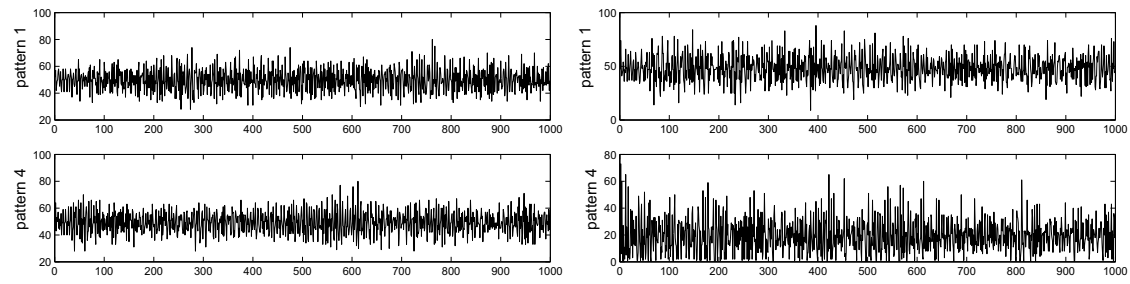

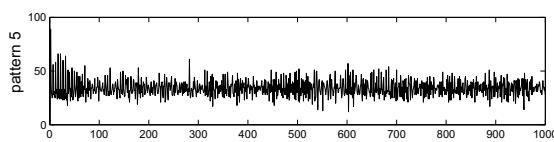

(a)

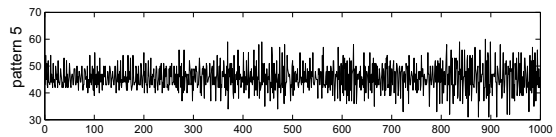

(b)

Fig. 3. The Hamming distance between the output and the trained patterns or untrained patterns while using different settings. The input is Pattern 4 in both (a) and (b). The external stimulation weights are $a_{i}=2$ and $a_{i}=2+6 x_{i}$ in (a) and (b) respectively. The output graph are displayed for pattern 1, pattern 4 and pattern 5 .

Table 1. The frequency distribution of the Hamming distance between the input and the output as the measure occurs within 10 intervals, when the external input is $a_{i}=2$, and the refractory factor $\alpha=10$.

\begin{tabular}{|c|c|c|c|c|c|c|c|c|c|c|}
\hline & \multicolumn{10}{|c|}{ Hamming distance } \\
\hline Input Pattern & {$[\mathbf{0 , 5}]$} & {$[\mathbf{6 , 1 9}]$} & {$[\mathbf{2 0 , 2 9}]$} & {$[\mathbf{3 0 , 3 9}]$} & {$[\mathbf{4 0 , 4 9}]$} & {$[\mathbf{5 0 , 5 9}]$} & {$[\mathbf{6 0 , 6 9}]$} & {$[\mathbf{7 0 , 7 9}]$} & {$[\mathbf{8 0 , 8 9}]$} & {$[\mathbf{9 0 , 1 0 0}]$} \\
\hline Pattern 1 & 1 & 3 & 25 & 129 & 279 & 397 & 143 & 22 & 1 & 0 \\
\hline Pattern 2 & 3 & 5 & 33 & 121 & 291 & 374 & 146 & 24 & 3 & 0 \\
\hline Pattern 3 & 1 & 2 & 15 & 117 & 342 & 358 & 146 & 18 & 1 & 0 \\
\hline Pattern 4 & 1 & 6 & 12 & 157 & 338 & 339 & 121 & 24 & 2 & 0 \\
\hline
\end{tabular}

the interval $[40,59]$ - i.e., almost $50 \%$ erroneous pattern, which is what we expect from a truly noisy pattern. If the same settings are used in the AdNN, the system behaves as an AM, which has been well illustrated by Adachi and his co-authors in their paper [1].

\subsection{Comparative PR Properties}

If the parameters of the AdNN are set as prescribed in [1], and the AdNN weights the external inputs with $a_{i}=2+6 x_{i}$, the system becomes closer to mimicing a PR phenomenon. Adachi et al claim that such a NN yields the stored pattern at the output periodically with a small periodicity. However, it turns out that if the input is an untrained pattern, the AdNN can still retrieve it and the other four trained patterns with relatively lower frequency. This property is more or less one which resembles an AM system instead of a PR system. This can be seen from Table 2 .

As opposed to this, for the L-AdNN, the most interesting scenario occurs when $\alpha=10$ and the weight for the external input is $a_{i}=2+6 x_{i}$. In this case, the system's output is noticeably chaotic. But the input pattern can be retrieved very frequently as long as the input is one of the trained patterns. 
Table 2. The AdNN: In this table, the input is the untrained Pattern 5 in Figure 2. The numbers in this table are the frequencies of each pattern being retrieved within the first 1,000 time intervals.

\begin{tabular}{|l|c|c|c|c|c|}
\hline & Pattern 1 & Pattern 2 & Pattern 3 & Pattern 4 & Pattern 5 \\
\hline Frequency & 44 & 0 & 1 & 34 & 110 \\
\hline
\end{tabular}

We clarify this by explaining Figure 3 (b) in greater detail. The input is Pattern 4, the output displays the input (Pattern 4) or a reasonably similar pattern with a very high frequency - as demonstrated by the low Hamming distance. The Hamming distance between the output and all the other patterns - the trained and the untrained pattern - is noticeably very large (i.e., with almost $50 \%$ noise).

The corresponding table of Hamming distances is given in Table 3. Consider the first column of Table 3 . The reader will observe that the trained input patterns can be approximately retrieved almost $20 \%$ of the time. On the contrary, when encountering an untrained input pattern (Pattern 5 in Figure 2), it is observed in the output with a much lower frequency - less than 5\%. Adachi and his co-authors did observe this for the AdNN, namely that the system with the external stimulations yielded a stored pattern with a relative higher frequency, and an untrained pattern with relative lower frequency - which phenomenon can be utilized to achieve PR. The amazing point here is that the L-AdNN displays the same phenomenon even though it merely has a linear number of connections, and the fact that the weights used are not directly obtained from the patterns themselves, but by migrating from the latter by using a gradient search algorithm. As far as we know, analogous properties have not been reported earlier for any type of NN (chaotic or otherwise). The unabridged paper [8] also presents a comparative analysis of the quasi-energy function properties of the L-AdNN and the AdNN, omitted here due to space considerations.

Table 3. The frequency distribution of the Hamming distance between the input and the output as the measure occurs within 10 intervals, when the external input is $a_{i}=2+6 x_{i}$ and the refractory factor $\alpha=10$.

\begin{tabular}{|c|c|c|c|c|c|c|c|c|c|c|c|c|}
\hline & \multicolumn{10}{|c|}{ Hamming distance } \\
\hline Input Pattern & {$[\mathbf{0 , 5}]$} & {$[\mathbf{6 , 1 9}]$} & {$[\mathbf{2 0 , 2 9}]$} & {$[\mathbf{3 0 , 3 9}]$} & {$[\mathbf{4 0 , 4 9}]$} & {$[\mathbf{5 0 , 5 9}]$} & {$[\mathbf{6 0 , 6 9}]$} & {$[\mathbf{7 0 , 7 9}]$} & {$[\mathbf{8 0 , 8 9}]$} & {$[\mathbf{9 0 , 1 0 0}]$} \\
\hline Pattern 1 & 217 & 342 & 226 & 112 & 63 & 28 & 10 & 2 & 0 & 0 \\
\hline Pattern 2 & 197 & 369 & 222 & 131 & 58 & 21 & 1 & 1 & 0 & 0 \\
\hline Pattern 3 & 198 & 325 & 251 & 130 & 57 & 32 & 3 & 4 & 0 & 0 \\
\hline Pattern 4 & 196 & 357 & 235 & 126 & 54 & 25 & 5 & 2 & 0 & 0 \\
\hline Pattern 5 & 46 & 495 & 334 & 104 & 18 & 2 & 0 & 1 & 0 & 0 \\
\hline
\end{tabular}

\section{Conclusions}

In this paper we have concentrated on the field of Chaotic Pattern Recognition (PR), which is a relatively new sub-field of PR. Such systems, which have only recently been investigated, demonstrate chaotic behavior under 
normal conditions, and resonate when it is presented with a pattern that it is trained with. The network which we have investigated is the Adachi Neural Network (AdNN) [1-5], which has been shown to possess chaotic properties, and to also demonstrate Associative Memory (AM) and Pattern Recognition (PR) characteristics. Because its structure involves a completely connected graph, the computational complexity of the AdNN is quadratic in the number of neurons. In this paper we have considered how the computations can be significantly reduced by merely using a linear number of computations. To achieve this, we have extracted from the original completely connected graph, one of its spanning trees and then computed the best weights for this spanning tree by using a gradient-based algorithm. Apart from a Lyapunov analysis and an analysis of the quasi-energy function found in [8], by a detailed experimental suite, we show that the new linear-time AdNN-like network possesses chaotic and PR properties for different settings.

\section{References}

1.M. Adachi and K. Aihara. Associative dynamics in a chaotic neural network. Neural Networks, 10(1):83-98, 1997.

2.M. Adachi and K. Aihara. Characteristics of associative chaotic neural networks with weighted pattern storage-a pattern is stored stronger than others. In The 6th International Conference on Neural Information Processing, Perth, Australia, volume 3, pages 1028-1032, 1999.

3.M. Adachi, K. Aihara, and M. Kotani. Pattern dynamics of chaotic neural networks with nearest-neighbor couplings. In Circuits and Systems, 1991, IEEE International Sympoisum on, Westin Stanford and Westin Plaza, Singapore, volume 2, pages 1180-1183, June 1991.

4.M. Adachi, K. Aihara, and M. Kotani. An analysis of associative dynamics in a chaotic neural network with external stimulation. In Proceedings of 1993 International Joint Conference on Neural Networks, Nagoya, Japan, volume 1, pages 409-412, 1993.

5.K. Aihara, T. Takabe, and M. Toyoda. Chaotic neural networks. Physics Letters A, 144:333-340, 1990.

6.D. Calitoiu, B.J. Oommen, and D. Nussbaum. Desynchronzing of chaotic pattern recognition neural network to model inaccurate parception. IEEE Transaction on Systems, Man, and Cybernetics-part B:Cybernetics, 37(3):692-704, 2007.

7.D. Calitoiu, B.J. Oommen, and D. Nussbaum. Periodicity and stability issues of a chaotic pattern recognition Neural Network. Pattern Anal Applic, pages 175-188, 2007.

8.Qin Ke and B. J. Oommen. Adachi-like chaotic neural networks requiring lineartime computations by enforcing a tree-shaped topology. In Unabridged Version of This paper, 2008.

9.Qin Ke and B. J. Oommen. Chaotic pattern recognition: The spectrum of properties of the adachi neural network. In The 12th International Workshop on Structural and Synaptic Pattern Recognition and 7th International Workshop on Statistical Pattern Recognition, December 2008. 